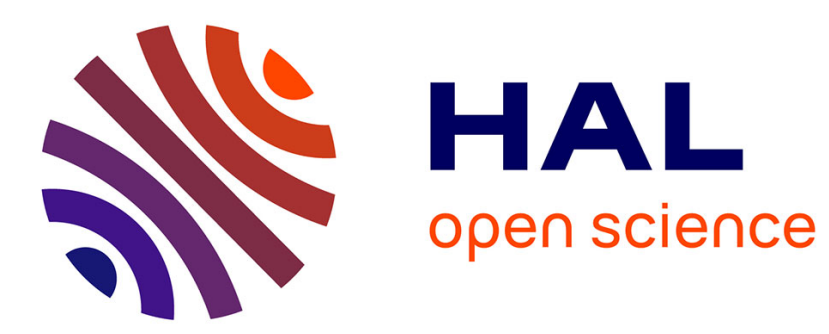

\title{
Spatially resolved optical characterization of functional materials using coherence scanning interferometry
}

Paul Montgomery, Rémy Claveau, Sébastien Marbach, Manuel Flury

\section{To cite this version:}

Paul Montgomery, Rémy Claveau, Sébastien Marbach, Manuel Flury. Spatially resolved optical characterization of functional materials using coherence scanning interferometry. physica status solidi (a), 2021, 2000683, 10.1002/pssa.202000683 . hal-03228227

\section{HAL Id: hal-03228227 \\ https://hal.science/hal-03228227}

Submitted on 4 Nov 2021

HAL is a multi-disciplinary open access archive for the deposit and dissemination of scientific research documents, whether they are published or not. The documents may come from teaching and research institutions in France or abroad, or from public or private research centers.
L'archive ouverte pluridisciplinaire HAL, est destinée au dépôt et à la diffusion de documents scientifiques de niveau recherche, publiés ou non, émanant des établissements d'enseignement et de recherche français ou étrangers, des laboratoires publics ou privés. 
DOI: doi.org/10.1002/pssa.202000683

\title{
Feature Article
}

\section{Spatially-resolved optical characterization of functional materials using coherence scanning interferometry}

Paul C. Montgomery*, Rémy Claveau, Sébastien Marbach and Manuel Flury

\author{
Dr. P. C. Montgomery
}

Laboratoire des Sciences de l'Ingénieur, de l'Informatique et de l'Imagerie (ICube), University of Strasbourg-CNRS-INSA,

23 rue du Loess, 67037 Strasbourg cedex, France

paul.montgomery@unistra.fr

Dr. R. Claveau

Laboratoire des Sciences de l'Ingénieur, de l'Informatique et de l'Imagerie (ICube), University of Strasbourg-CNRS-INSA,

300 Boulevard Sébastien Brant, 67412 Illkirch cedex, France

Mr. S. Marbach

Laboratoire des Sciences de l'Ingénieur, de l'Informatique et de l'Imagerie (ICube), University of Strasbourg-CNRS-INSA,

300 Boulevard Sébastien Brant, 67412 Illkirch cedex, France

Dr. M. Flury

Laboratoire des Sciences de l'Ingénieur, de l'Informatique et de l'Imagerie (ICube), University of Strasbourg-CNRS-INSA,

300 Boulevard Sébastien Brant, 67412 Illkirch cedex, France

Keywords: local spectroscopy, surface metrology, materials characterization, interference microscopy, Linnik interferometer

We review some of our work in adapting white light interference microscopy to perform local spectroscopy for measuring the optical properties of microscopic structures. Theoretical and experimental results are shown in which Fourier transform processing of the polychromatic 
fringe signal combined with careful calibration of the optical system is used to make measurements of local reflectance spectra. This approach captures the spectral information within the entire field of view in a single scan, allowing rapid spectral mapping of spatially extended surfaces. Results are shown of local reflectance spectra measured on different materials and buried under transparent layers with a lateral spot size of between $0.5 \mu \mathrm{m}$ and several $\mu \mathrm{m}$ over a field of view up to $650 \times 650 \mu \mathrm{m}$. The technique is extended to the measurement of local refractive index and thickness of transparent layers as well as to the size determination of small spherical beads buried in transparent and scattering layers with a priori information. The significance of the work is the potential for local materials characterization in complex, hybrid and functional materials and even disease detection through the study of living cells.

\section{Introduction}

The use of functionalized and complex surfaces and layers promises to lead to new functional materials with novel properties such as for example being able to detect and react to local stimuli or to detect biological properties. To achieve such functions, a growing challenge exists to characterize the physical properties of the individual microscopic components. Classical spectroscopy for measuring optical properties typically uses a large probing area over several $\mathrm{mm}^{2}$. Recently, new techniques based on near field optical microscopy and hyperspectral imaging have been developed to perform spectral measurements on a local scale, in which the examination of large areas requires scanning in either the spatial or spectral domains.

For performing s-SNOM (scattering-type scanning near-field optical microscopy) for example, Huber et al. ${ }^{[1]}$ use a commercial metalized Si tip in a custom-built AFM system to serve as topographical and near-field optical (s-SNOM) probe simultaneously. Illumination of the tip with the focused beam of a $\mathrm{CO}_{2}$ laser provides mid-IR frequencies between 885 and $1085 \mathrm{~cm}^{-1}$ for local spectroscopic characterization and the elastically scattered light from the tip is detected simultaneously to provide topographic measurements. The combination of vertical tip resonant oscillation at $300 \mathrm{kHz}$ and pseudo-heterodyne interferometric detection yields very low noise amplitude $s_{n}$ and phase $\phi_{n}$ images. Their results demonstrate the potential of s-SNOM for infrared spectroscopic recognition of materials within individual semiconductor nanodevices with a spatial resolution of $20 \mathrm{~nm}$, for example in the mapping of amorphous $\mathrm{SiO}_{2}$ and $\mathrm{Si}_{3} \mathrm{~N}_{4}$ components in transistors fabricated in $65 \mathrm{~nm}$ technology. Amenabar et al. ${ }^{[2]}$ use the same principle of s-SNOM to perform what they call nano-FTIR for 
the mapping of protein structure with $30 \mathrm{~nm}$ lateral resolution and sensitivity to individual protein complexes. The disadvantages of such near-field imaging is the need for pointwise scanning and measuring the spectral response at each pixel with a separate spectrometer which makes the instrumentation complex and mapping measurement time consuming. Hyperspectral imaging microscopy enables a set of images to be obtained of the same field of view with a full field optical microscope in which each image is taken over a narrow spectral band. It is possible to either scan laterally and take the spectra at each pixel ${ }^{[3]}$ or to scan the wavelength of the light source for complete images. This results in a hyperspectral cube with the lateral dimensions over XY and the spectral information over Z. For example, in ${ }^{[4]}$, an enhanced commercial dark field transmission optical microscope (Olympus BX41) is used, equipped with a hyperspectral imaging spectrophotometer (CytoViva) to measure the spectra at each pixel of different types of engineered nanoparticles $\left(\mathrm{Ag}, \mathrm{TiO}_{2}, \mathrm{CeO}_{2}\right.$ for example) under $50 \mathrm{~nm}$ in size in wet environmental systems. Spectral libraries made from calibrated samples in ultrapure water are used to determine the presence of nanoparticles in unknown liquid samples. While the spectral resolution is very good because of the use of a spectrometer, the need for XY scanning lengthens the acquisition time for a single image measurement to several minutes.

In the field of optical metrology, interference microscopy is now a well-known technique for the measurement of surface roughness and microscopic 3D structures. ${ }^{[5-7]}$ By adding a color camera to an interference microscope it is possible to perform a simple type of qualitative local spectroscopy using the RGB signature at each point in the image. ${ }^{[8]}$ While this allows basic material identification, because it is not quantitative, it is not able to distinguish between two similar looking or metamer colored materials. ${ }^{[9]}$

Quantitative local spectroscopy can be performed with white light interference microscopy by using Fourier transform processing of the polychromatic fringe signal combined with careful calibration of the optical system. ${ }^{[10-12]}$ This approach captures the spectral information within the entire field of view in a single scan, with the advantages of allowing rapid spectral mapping of spatially extended surfaces.

In this paper, we present a short review of the work we have carried out in this field since 2016 in order to extend local spectroscopy measurements using interference microscopy from local structures on just surfaces to those buried in transparent ${ }^{[13]}$ and scattering layers. ${ }^{[14]}$ First, the basic principles of local spectroscopy using interference microscopy are presented, with some examples of spectroscopic measurements of different materials in air, followed by an explanation of spatially resolved (XYZ) local spectroscopy with a lateral resolution of a few 
$\mu \mathrm{m}^{2}$ over an area up to $650 \times 650 \mu \mathrm{m}$ and an axial resolution of about $3.5 \mu \mathrm{m}$ through polymer material. Then a technique for performing local measurements of refractive index and thickness of transparent layers is presented. Finally, the characterization of individual spherical beads buried in transparent and scattering layers is described.

\section{Basic principles of local spectroscopy using interference microscopy}

Interference microscopy is now a well-known technique used in optical metrology for the measurement of surface roughness and microscopic 3D structures. ${ }^{[5-7]}$ It is also becoming more widely used in the tomographic analysis of transparent materials and layers. ${ }^{[15,16]}$ The main principle behind the techniques is the use of white light interference fringes as a probe in an optical microscope to determine the position of a surface or sub-surface structures to be measured at each point in the image. Since polychromatic light is used for surface measurements, it is also possible to carry out local spectroscopic measurements with the same system by applying a Fourier transform to the interference fringes. This leads to information about the source spectrum, the spectral response of the optical system and the reflectance spectrum of the surface. ${ }^{[13,14]}$

The general design for the Linnik interferometer is shown in Figure 1(a), consisting of two interference arms, one with a reference mirror and the other the sample surface. The two interference microscopes used in the present work consist of a modified Leitz-Linnik ${ }^{[13]}$ and a home built breadboard Linnik, as shown in Figure $1(\mathrm{~b}) .{ }^{[17]}$ In the Leitz-Linnik the illumination source used was an incandescent lamp with an $800 \mathrm{~nm}$ central wavelength and a $290 \mathrm{~nm}$ bandwidth and the camera was a Basler avA1000-100gc GigE or a Photonfocus MV1-D2048I-96G2-10, having a spectral sensitivity range between $500 \mathrm{~nm}$ and $700 \mathrm{~nm}$ for the former and from $500 \mathrm{~nm}$ to $1100 \mathrm{~nm}$ for the latter.

In the breadboard Linnik, the light source consisted of a white LED with a center wavelength of $660 \mathrm{~nm}$ and a bandwith of $380 \mathrm{~nm}$ and the camera used was a 16 bits peltier cooled PCO Edge 4.2 USB3.0 model with an sCMOS sensor, providing a spectral sensitivity range from $500 \mathrm{~nm}$ to $800 \mathrm{~nm}$. This system uses Köhler illumination with control of the illumination properties using two diaphragms (field and aperture). The Leitz-Linnik uses two x50 microscope objectives (quoted NA=0.85) and the breadboard system uses two x20 longworking distance objectives $(\mathrm{NA}=0.42)$. Both systems are controlled with a lab-developed LabVIEW program. 


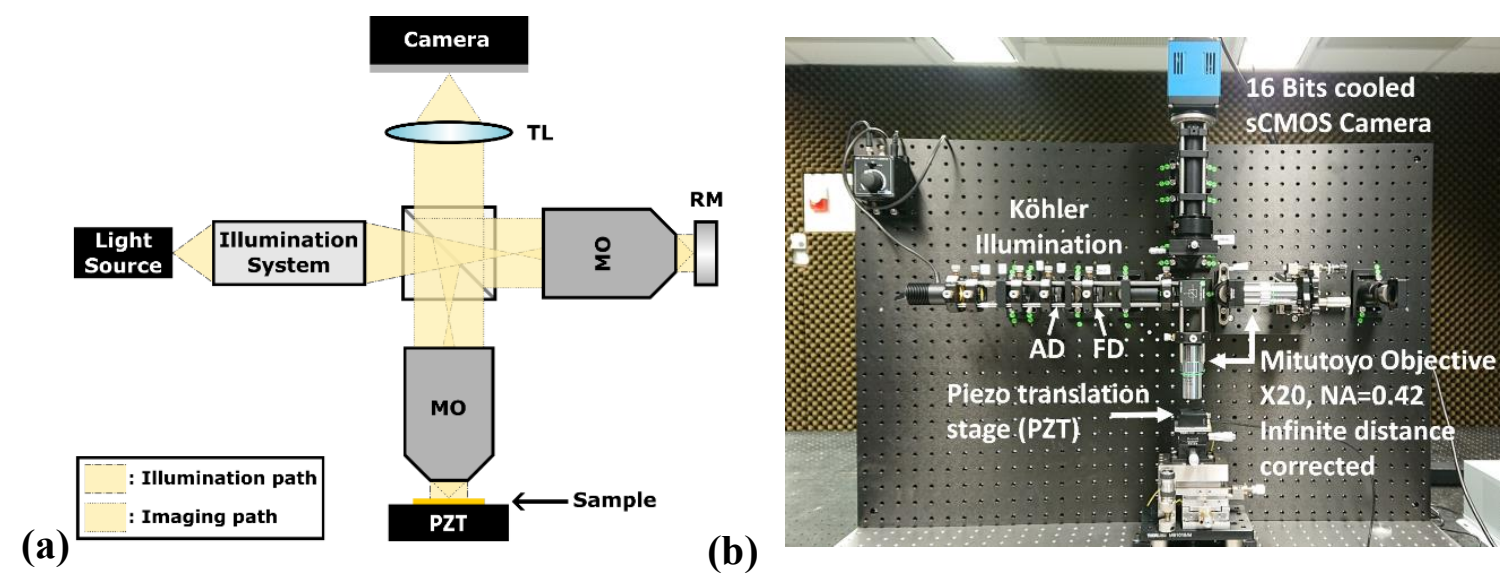

Figure 1 Linnik interference microscope system used for local spectroscopy (a) schematic diagram of the optical system; TL, tube lens $\mathrm{f}=200 \mathrm{~mm}$; MO, microscope objectives; RM, reference mirror; PZT, piezoelectric device, (b) experimental breadboard system with Mitutoyo long distance objectives with large field of view; A.D, aperture diaphragm; F.D, field diaphragm. Reproduced with permission, from S. Marbach, C. Cordier, R. Claveau, T. Engel, P.C. Montgomery, M. Flury, White light interference microscopy system design, Proceedings of SPIE Photonics Europe Digital Forum, Photonics and Plasmonics at the Mesoscale, Vol 11352, 2020. ${ }^{[17]}$

The light coming from the source is divided into two beams by the beamsplitter, to travel into the reference and the object arms. The reference and object beams are then respectively reflected by a reference mirror and the sample and superimposed by travelling again through the beamsplitter to form the interference image on the camera. The sample is placed on a piezo-translation stage to allow nm-sensitive movement along the optical axis.

\subsection{Theory for local spectroscopy using interference microscopy}

After scanning the fringes over $\mathrm{Z}$, such an interferometer gives a typical set of white light fringes as shown in Figure 2. ${ }^{[13]}$

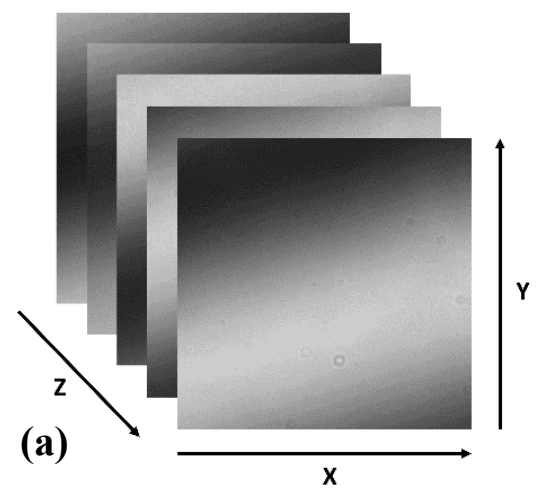

(b)

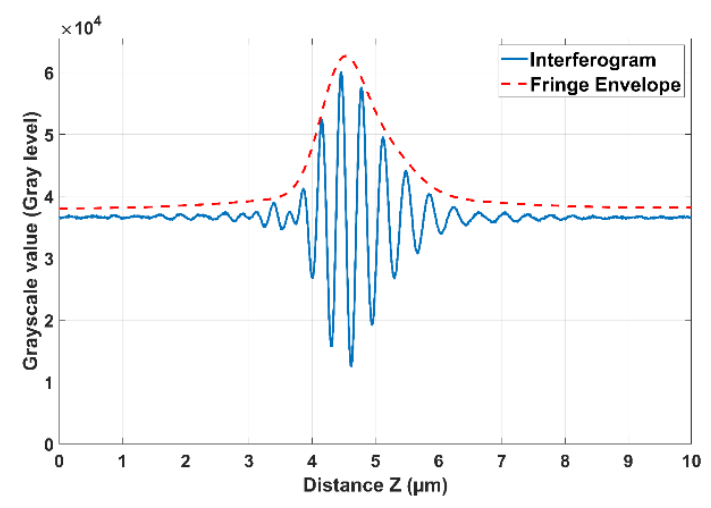


Figure 2 Typical results of interference fringes from an interference microscope after scanning over $\mathrm{Z}$ (a) an XYZ stack of sampled fringe images, (b) a typical signal profile at a single pixel.

Let us consider an interferometric signal representing the intensity profile along the optical axis $\mathrm{Z}$ at a given pixel of the XYZ image stack as shown in figure 2(b). ${ }^{[13]}$

The plane wave emitted by the light source is denoted by $E(k, t, z)=s(k) \mathrm{e}^{\mathrm{j}(\omega t-k z)}$, where $s(k)$ is the amplitude of the source to the frequency $k$. After propagation through the interferometer and reflection on the reference mirror and the sample surface, the interference of these two electric fields at the camera results in a given intensity. Since the illumination source has a broadband spectrum, the contributions of all the different wavelengths must be taken into account. Polarization is not considered in this particular optical setup. Only the rays of the same wavelength can interfere with each other, resulting in a total intensity which is the sum of the interferences at each wavelength:

$$
I(z)=\int_{0}^{+\infty} S(k)\left\{\frac{1}{4}\left[R_{\text {ref }}(k)+R_{s}(k)\right]+\frac{1}{2} \sqrt{R_{r e f}(k)} \sqrt{R_{s}(k)} \cos (\Delta \varphi(k, z)+\phi)\right\} d k,
$$

$R_{\text {ref }}(k)$ and $R_{s}(k)$ are respectively the intensity reflection coefficients of the reference mirror, and the sample, $S(k)$ is the power spectral density of the source, $\Delta \varphi(k, z)$ denotes the phase shift between the two waves, and $\phi$ represents the phase shift introduced by the reflection.

\subsection{Spectral analysis of the interferometric signal}

The spectral analysis is performed by applying a Fourier transform to the interferometric signal given in Equation (1). This technique allows the recovery of the spectral properties of the surface under study as well as those of the entire optical system. ${ }^{[10]}$ The effective spectrum of the system is designated by $S_{\text {eff }}(k)=S(k) \sqrt{ } R_{\text {ref }}(k) \sqrt{ } R_{s}(k)$ and the phase shift is given by $\Delta \varphi(k, z)=2 k z$. The intensity signal at the detector can also be written as:

$$
I(z)=A+\frac{1}{2} \operatorname{Re}\left\{\operatorname{TF}^{-1}\left[S_{\text {eff }}(k) e^{j \phi}\right]\right\} \text { where } A=\int_{0}^{+\infty} \frac{S(k)}{4}\left[R_{\text {ref }}(k)+R_{s}(k)\right] d k .
$$

$A$ is the mean value of the signal and $\phi$ is still the phase shift introduced by reflection. After removing $A$, the Fourier transform is applied and the modulus taken:

$$
|\operatorname{TF}[I(z)]|=\left|\frac{1}{2} S_{\text {eff }}(k) e^{j \phi}\right|=\frac{1}{2} S_{\text {eff }}(k)=\frac{1}{2} S(k) \sqrt{R_{\text {ref }}(k)} \sqrt{R_{s}(k)} .
$$

In practice, however, the measured effective spectrum accounts for the spectral sensitivity of the sensor as well as for the transmissivity of all the optical components, which leads to the Non-Calibrated Spectral response (NCS): 
$\operatorname{NCS}(k)=S(k) R_{\text {sys }}(k) \sqrt{R_{\text {ref }}(k)} \sqrt{R_{s}(k)}$,

where $R_{\text {sys }}(k)$ includes the spectral response of the camera and the spectral transmissivity of the optical system.

Performing a reflectance measurement then requires two spectral measurements. The first one is performed on a sample of known reflectance denoted by $R_{s}(k)=R_{c a l}(k)$ and is usually measured with a spectrometer. This measurement, referenced as the calibration step, makes it possible to deduce the spectral properties of the system. Following this step it is possible to determine experimentally the Spectral Transfer Function (STF)":

$\operatorname{STF}(k)=\frac{N C S(k)}{\sqrt{R_{c a l}(k)}}=S(k) R_{s y s}(k) \sqrt{R_{r e f}(k)}$

The second measurement is performed on the sample under study. By taking care to make both measurements in the same experimental conditions (in terms of the intensity of the source, the surrounding influences and the optical arrangement), the reflectance of the surface is simply obtained using the following equation:

$$
R_{s}(k)=\left|\frac{\operatorname{TF}[I(z)]_{s}}{\operatorname{TF}[I(z)]_{c a l}}\right|^{2} R_{c a l}(k)
$$

The results in Figure 3 (a) show examples of simulated STF obtained by Fourier transforming a synthetic interferogram generated from a perfectly reflective surface, i.e., a surface that reflects $100 \%$ of the incident light for all the wavelengths within the source spectrum, for different objective numerical apertures. 
(a)

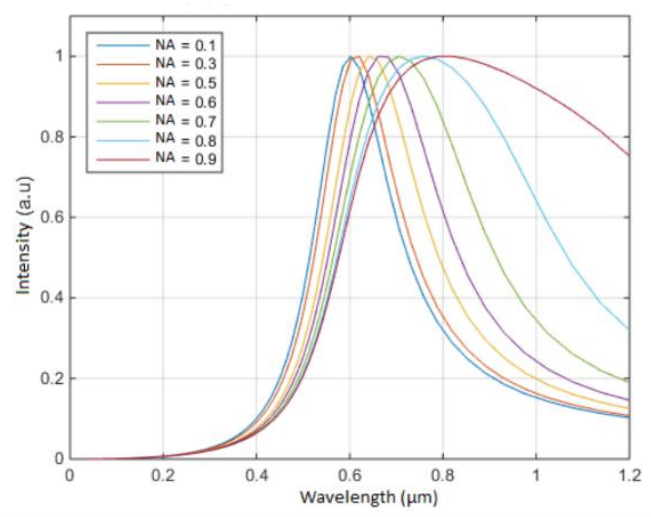

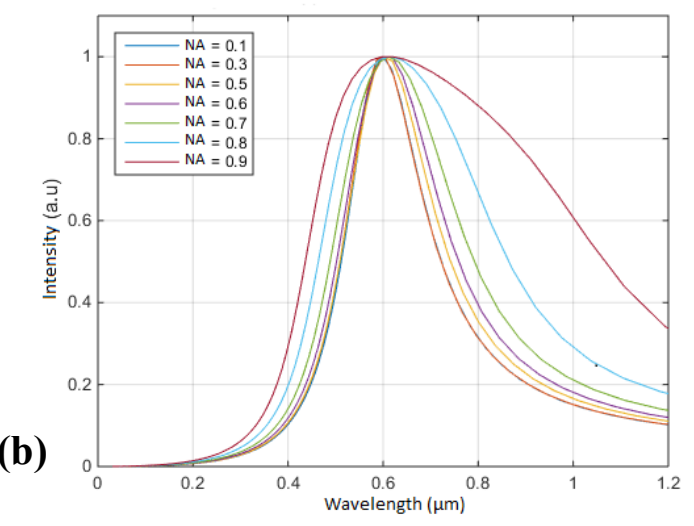

(b)

(c)

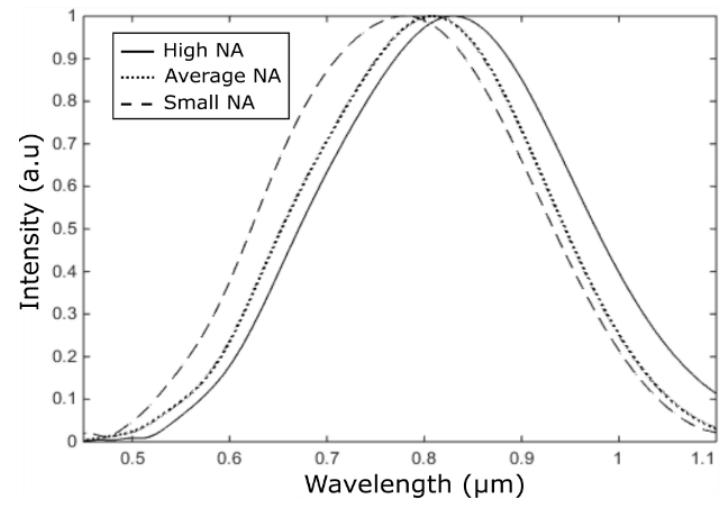

Figure 3 Set of simulated and measured STF. Simulated transfer function for different NA of the objectives before (a) and after correction (b). (c) Measured transfer function of the Leitz Linnik microscope for different system apertures (the same objective NA is used but the illumination aperture varies). Reproduced with permission, from R. Claveau, P. Montgomery, M. Flury, D. Montaner, Local reflectance spectra measurements of surfaces using coherence scanning interferometry, Proceedings of SPIE Photonics Europe: Optical Micro- and Nanometrology, Vol 9890, 2016. ${ }^{[13]}$

These results show that the reconstructed effective spectrum widens and is shifted towards longer wavelengths when illuminating at oblique angles. This phenomenon is also observed on experimental measurements in Figure 3 (c) for which the $S T F$ was measured by Fourier transforming an interferogram captured on a silicon wafer for different openings of the aperture diaphragm, which results in a slight change of the global system aperture. For spectral characterization, this aperture effect needs to be corrected to avoid measurement errors and to improve the precision of the spectral measurements. A correction factor, called the $\beta$-factor, has been introduced ${ }^{[13,18]}$ to reverse the widening and shifting effects observed at large apertures. However, as shown in Figure 3(b), this simple correction is not sufficient for correcting all the errors introduced at high NAs. While slightly decreasing the lateral resolution, we found that closing the aperture diaphragm was a better approach and led to better results in terms of spectral measurement accuracy. 


\subsection{Results of local spectroscopy on different materials}

Some results of experimental local spectroscopic measurements on two different materials are shown in Figure 4. The reflectance values are given for the local method (blue line) as a function of the wavelength. The first (Figure 4(a)) was obtained using the Leitz-Linnik interferometer with a standard CCD camera on a thin layer of doped tin oxide $\left(\mathrm{SnO}_{2}\right)$ deposited on a silicon substrate. ${ }^{[13]}$ The local measurements, made over an area of $0.58 \mu \mathrm{m}^{2}$ (limited by diffraction), are compared with the results obtained from a conventional optical spectrometer that uses a spot size of several $\mathrm{mm}^{2}$ (black line). It can be observed that there is an increase in the uncertainty (standard deviation) as well as a slight deviation from the spectrometer results around the extreme limits of the measured spectral range (around $450 \mathrm{~nm}$ and above $600 \mathrm{~nm}$ ). This is a direct consequence of the processing since the reflectance is calculated by dividing the NCS by the $S T F$. Because the $S T F$ falls off for lower signal intensities, consequently the measurement uncertainty increases rapidly for the wavelengths at which the STF falls near to 0 . The second set of results (Figure 4(b)) were made on a layer of Au on Si using the breadboard Linnik with the cooled camera (Figure 1(b)). The local measurements (blue and red lines) are compared with those from simulations using the Fourier modal method using the gold dispersion (black line). To reduce the noise and the uncertainties in the spectral measurements, both temporal averaging and spatial filtering are used. For example, in Figure 4(b), 10 successive image stacks were averaged and the blue curve was spatially filtered over an area of 10x10 pixels, corresponding to a measurement spot size of $3.25 \times 3.25 \mu \mathrm{m}$ and at the expense of a longer processing time.

(a)

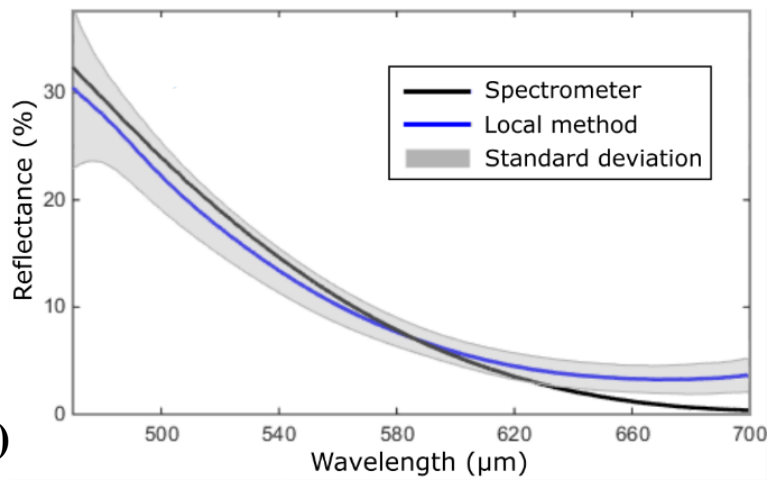

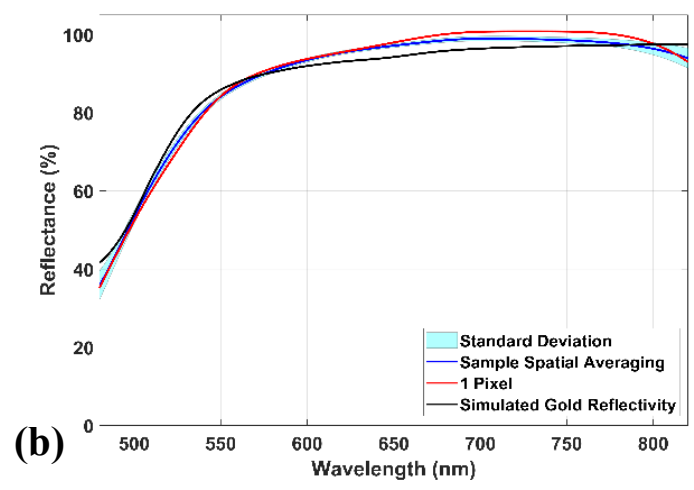

(b)

Figure 4 Mean value and standard deviation of the measured reflectance spectrum for (a) thin layer (hundreds of $\mathrm{nm}$ ) of doped tin oxide deposited on Si using the Leitz-Linnk microscope. Reproduced with permission, from R. Claveau, P. Montgomery, M. Flury, D. Montaner, Local reflectance spectra measurements of surfaces using coherence scanning interferometry, Proceedings of SPIE Photonics Europe: Optical Micro- and Nanometrology, Vol 9890, 
$2016 .^{[13]}$ (b) gold layer deposited on Si using the breadboard Linnik system shown in Fig. 1(b).

\section{Spatially (XYZ) resolved local spectroscopy using interference microscopy}

Having developed the basic theory and technique for performing local spectroscopy at a single point on a sample with interference microscopy, the next step is to investigate the various aspects concerning the spatial resolution, both in the lateral plane of the image, XY and over the depth, Z, in transparent samples.

\subsection{Results on multiple material test sample in the same field of view}

One main application of the local spectroscopy technique is to be able to study different materials on the same sample and in the same field of view. With this in mind, we developed a sample consisting of four different materials with the surfaces roughly in the same plane ${ }^{[19}$,

${ }^{20]}$ that could be measured locally using the proposed technique as well as with a classical spectrometer with a larger spot size.

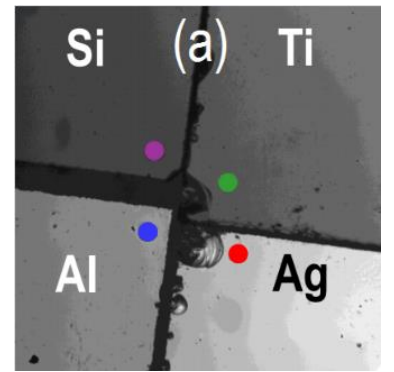

(a)

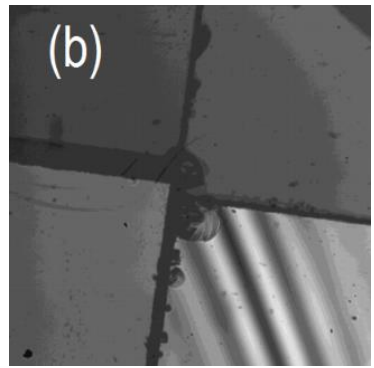

(b)

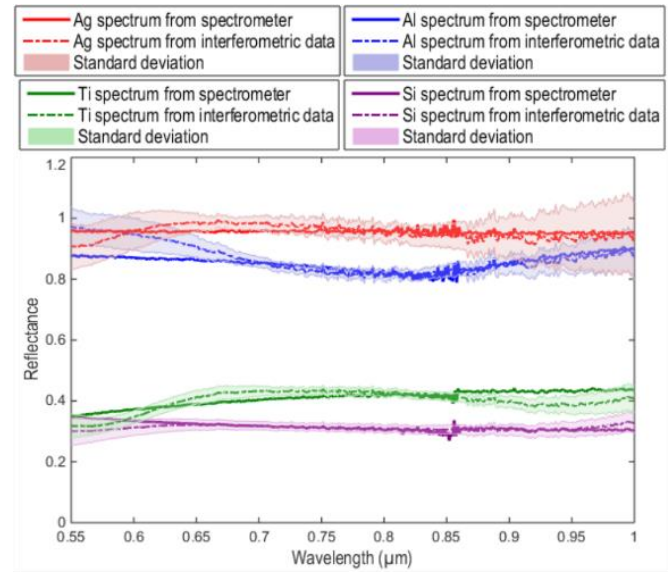

(c)

Figure 5 Local spectroscopy measurements of 4 different materials ( $\mathrm{Si}, \mathrm{Ti}, \mathrm{Al}, \mathrm{Ag}$ ) on same sample (a) direct optical image of the sample, (b) interference fringes on the Ag material extracted from the stack, (c) reflectance spectra of the four materials measured using the local interferometric method (dashed lines) and an optical UV-VIS-IF spectrometer (solid lines). The image size is $1.19 \times 1.19 \mathrm{~mm}^{2}$. Reproduced with permission, 2017, Wiley. ${ }^{[19]}$

The four well known materials are a silicon ( $\mathrm{Si}$ ) wafer, a piece of polished titanium (Ti), an aluminum (Al) layer evaporated onto silicon and a silver (Ag) layer evaporated onto a silicon wafer (Figure 5(a)). The Leitz-Linnik interferometer was used to perform the local 
spectroscopy tests, Figure 5(b) showing interference fringes on the silver part of the sample. Fringes cannot be observed everywhere since the upper surfaces were not exactly in the same plane but not far from each other.

The local interferometric spectroscopy technique described previously was then used at four points, one in each of the middle of the four different materials in the same field of view. An optical UV-VIS-IF spectrometer (Perkin-Elmer Lambda 19 UV-VIS-NIR) was then used to measure the same materials with the larger spot size of several $\mathrm{mm}^{2}$. The results of the locally measured reflectance spectra of the four materials after processing (dashed lines) and the spectrometer (solid lines) are shown in Figure 5(c). It can be seen that there is very good correspondence with the measurements of the classical spectrometer. The explanation of the discrepancies between the results of the local technique and those of the spectrometer (especially for the $\mathrm{Al}$ measurement) is the same as that stated in the previous section. The uncertainty increases due to a decrease in the signal to noise ratio for the extreme wavelengths at which the $S T F$ starts falling off. Because the results of the local spectroscopic technique are averaged spatially over an area of $5 \times 5$ pixels to reduce noise, the lateral measurement spot size is about $0.5 \mu \mathrm{m}$ in diameter. While these measurements have been validated for flat surfaces, for samples having local slope or curvature, it would be expected that there would be a modification in the effective spectrum ${ }^{[21]}$ that would require the development of a correction strategy.

\subsection{Depth resolved local spectroscopy using interference microscopy}

Having demonstrated the successful measurements of the local spectroscopic technique on different materials in the same field of view, the next step is to test the capabilities of the technique over depth in a transparent sample. This is possible since separate packets of white light fringes are formed along the optical axis at the same point due to reflections at the air/layer surface and the layer/substrate interface (Figure 6(a)). ${ }^{[19]}$ If these are well separated, then the signal can be windowed along $\mathrm{Z}$ and the local spectroscopy technique applied to each interface.

In order to test this idea, a suitable test sample was made consisting of a PMMA (polymethyl methacrylate) layer of several $\mu \mathrm{m}$ thickness made by spin coating on a silicon substrate on which some small silver squares had been deposited so as to provide two different buried materials. The sample was then scanned over $\mathrm{Z}$ on the Leitz-Linnik interferometer. The previously described technique of applying a Fourier transform to the corresponding windowed interferometric signals and using an accurate calibration of the optical system was then used to determine the reflectance spectra of the silicon and silver materials buried under 
the PMMA layer. A comparison of the measurements with those from simulation using the electromagnetic matrix S-method for stratified media showed a very good correspondence (Figure 6(b)).

(a)

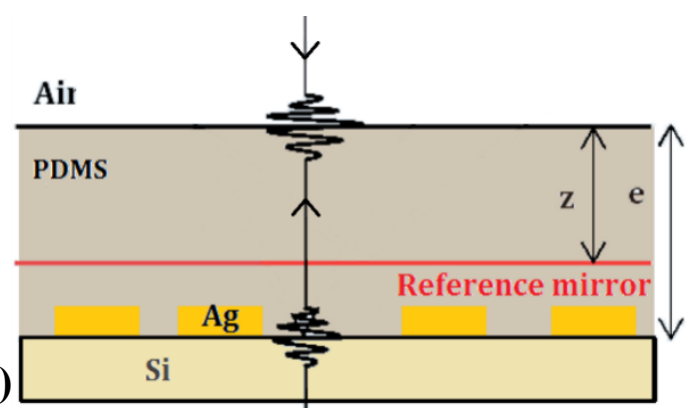

(b)

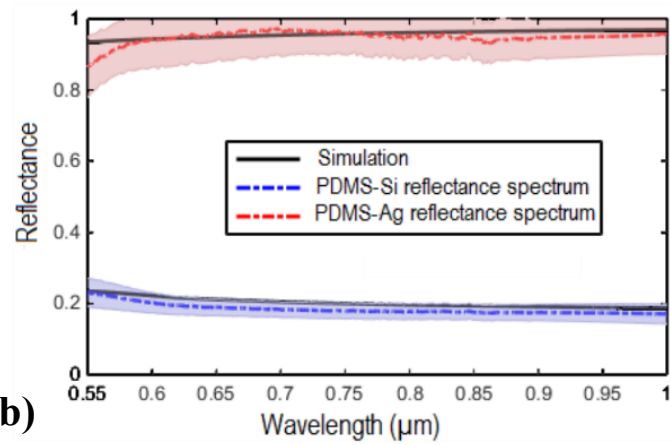

Figure 6 Local spectroscopy through a transparent layer, (a) schematic representation of the layer of PDMS deposited on a substrate of silicon with deposited silver dots and the interferometric signal along the Z-axis. Reproduced with permission, from R. Claveau, P. Montgomery, M. Flury, Proceedings of SPIE Photonics Europe, Optical Micro- and Nanometrology, Vol 10678, 2018. ${ }^{[27]}$ (b) depth-resolved reflectance spectra of a layer of Ag and PDMS-Si buried interfaces. The experimental results are compared to simulations (black lines). Both were obtained by working at normal incidence. Reproduced with permission, 2017, Wiley. ${ }^{[19]}$

In this case, working in normal incidence (the aperture diaphragm AD is closed) is essential. Failure to reduce the angle of the illumination cone results in degraded spectral measurements as their amplitude is severely attenuated. ${ }^{[18,20]}$ Because of the need to close down the AD, the lateral measurement spot size is increased to $0.85 \mu \mathrm{m}$ in diameter. These experimental results demonstrate that depth-resolved reflectance spectra of buried materials can be performed with interference microscopy.

\section{Local measurements of refractive index and thickness of transparent layers}

Since the developed technique provides local spectroscopic information, this can also be used to measure the local refractive index and thickness of transparent layers using either the magnitude or the phase of the thin film total reflectance spectrum. Two cases can be considered. The first one is that of very thin films which have an optical thickness (product of the real thickness with the optical index) that is lower than the coherence length of the light source used. Here, the multiple reflections of the light within the layer have to be taken into account and the magnitude of the FT corresponds then to the well-known total reflection coefficient given by: 
$r_{\text {tot }}(k)=\frac{r_{s_{1}}+r_{s_{2}} \exp (-2 j k e n \cos \theta)}{1+r_{s_{1}} r_{s_{2}} \exp (-2 j k e n \cos \theta)}$

where the terms $r_{s 1}$ and $r_{s 2}$ are the Fresnel reflection coefficient of the front and rear interfaces of the layer, $e$ and $n$ are its thickness and refractive index and $\theta$ the propagation angle within the layer.

The second case, which is of more interest here, is that of thicker layers having an optical thickness that exceeds the coherence length of the light source used, being typically more than $1 \mu \mathrm{m}$. Various microscope techniques have been developed for measuring the local thickness of thin transparent layers of up to a few $\mu \mathrm{m}$ in thickness that use either separate spectrometers connected to the microscope,${ }^{[22,23]}$ or direct frequency domain analysis of the fringes within the microscope interferometer. ${ }^{[24,25]}$ Here the light propagating more than one round trip within the layer can be neglected since its optical path is much higher than that being reflected only once. In this case, a new model must be established for the spectral response of the transparent layer. The following step in the present work was thus to develop techniques for the local characterization of transparent materials with thicknesses ranging from a few hundred nanometers to several $\mu \mathrm{m}$.

Neglecting $2^{\text {nd }}$ order reflections, applying a Fourier transform to the interference signal from the transparent layer and taking the magnitude squared leads to the expression of the spectral reflectance of the layer and can be written as: ${ }^{[26]}$

$\left|r_{\text {tot }}(k)\right|^{2}=r_{s_{1}}^{2}(k)+r_{s_{2}}^{2}(k)\left(1-r_{s_{1}}^{2}(k)\right)^{2}+2 r_{s_{1}}(k) r_{s_{2}}(k)\left(1-r_{s_{1}}^{2}(k)\right) \cos \left(2 k e n \cos \theta+\phi_{1}-\phi_{2}\right),(8)$

The spectral reflectance $\left|r_{t o t}(k)\right|^{2}$ denotes the model developed in order to recover the properties of the layer and $r_{s i}(k)$ and $\phi_{i}$ refer to the magnitude and phase of the Fresnel reflection coefficient of the interface i (1: air/layer, 2: layer/air). This expression assumes that no absorption occurs during the light propagation.

The local thickness and refractive index can then be retrieved by finding the best fit between the experimentally measured spectrum and the theoretical model using a non-linear leastsquares algorithm. ${ }^{[27]}$ Two samples were prepared to make experimental measurements and to demonstrate the proposed technique. The first consisted of a transparent PMMA layer spincoated onto a silicon substrate. The second was a layer of $\mathrm{SnO}_{2}$ on silicon which was more dispersive, made using reactive magnetron sputtering (AJA ORION 3 system) at $100{ }^{\circ} \mathrm{C}$ with a tin target. The results of the $\mathrm{SnO}_{2}$ layer are shown in Figure 7. The first image (Figure 7(a)) shows simulated fringes containing double fringe envelopes from the front and rear surfaces of a transparent layer $(e=1500 \mathrm{~nm}, n=1.484)^{[27]}$ to illustrate the type of fringes dealt with and to develop the model for calculating the theoretical dispersion response. 
After scanning the samples on the Leitz-Linnik microscope, a Fourier transform is applied to the windowed fringe packet. It is important to note that the aperture diaphragm of the illumination system needs to be closed so as to reduce the errors in the amplitude of the measured spectrum and achieve correct measurements of the refractive index. If the refractive index is assumed to be constant, the resulting model is slightly quicker to calculate but the match between experimental and theoretical reflectance spectra is not sufficient, in particular for cases where the dispersion of the layer is significant. On the other hand, by taking a wavelength-dependent index, for example using one defined by Sellmeier's law, the match is much improved. The results for the $\mathrm{SnO}_{2}$ layer in Figure 7(b) show a comparison between the experimental reflectance spectrum (black curve) and the theoretical optimized model (blue curve) with $n$ taken as a function of the wavelength. ${ }^{[26]}$ The resulting dispersion law over a wavelength range of 600-1000 $\mathrm{nm}$ in Figure 7(c) shows the average refractive index of 10 measurements (blue curve) and the standard deviation (grey area). The comparison with measurements using ellipsometry (dashed-dotted line) shows that the results are quite satisfactory even for a highly dispersive layer.

(a)
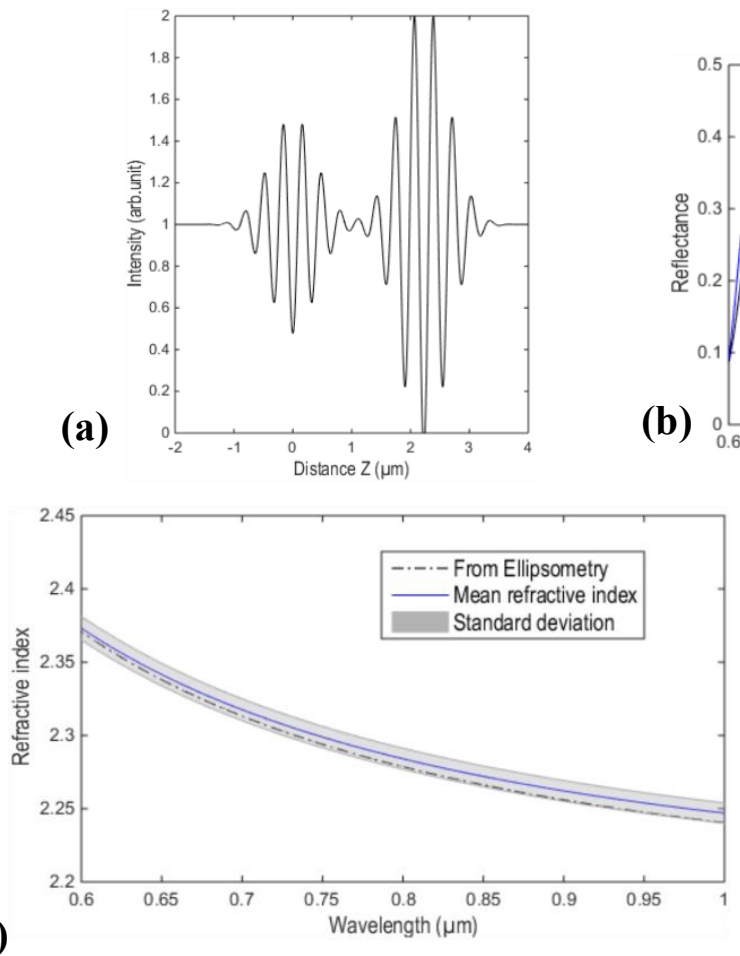

(b)
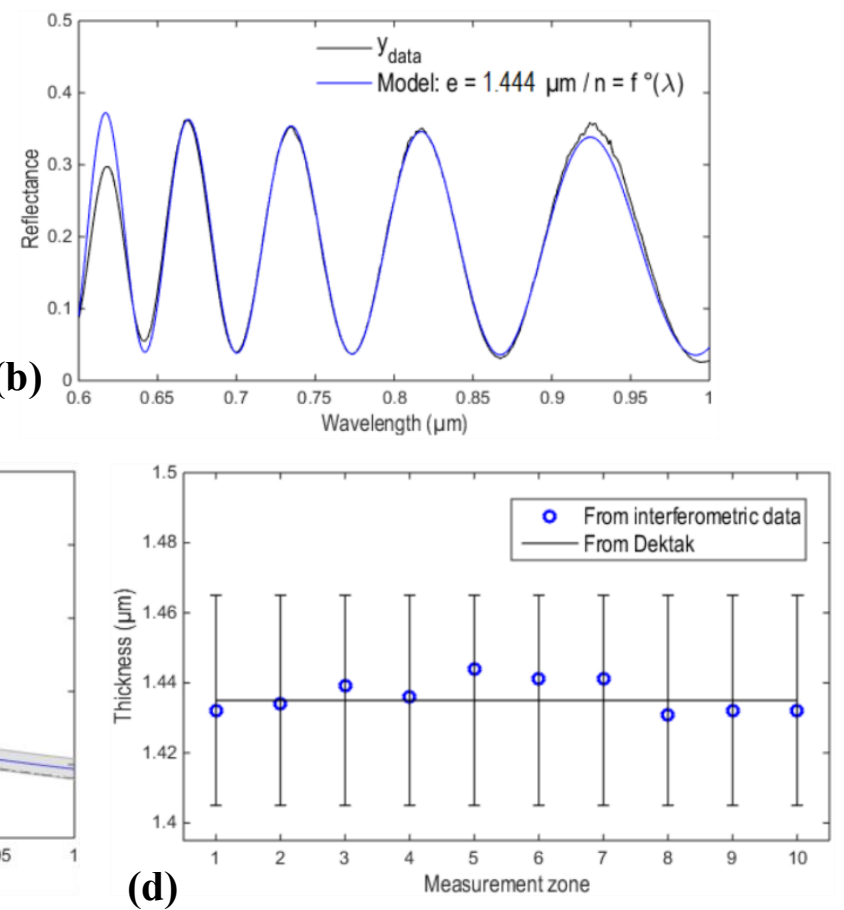

Figure 7 Use of interference microscopy to measure local thickness and refractive index of a dispersive layer of $\mathrm{SnO}_{2}$ on a $\mathrm{Si}$ substrate (a) simulated fringes showing double fringe envelopes from the front and rear surfaces of a transparent layer. Reproduced with permission, from R. Claveau, P. Montgomery, M. Flury, Application of coherence scanning interferometry for local spectral characterization of transparent layers, Proceedings of SPIE Photonics Europe, Optical Micro- and Nanometrology VII, Vol 10678, 2018. ${ }^{[27]}$ (b) 
comparison between the experimental reflectance spectrum (black curve) and the theoretical optimized model (blue curve) with $n$ taken as a function of the wavelength. Reproduced with permission, 2018, Elsevier, ${ }^{[26]}$ (c) dispersion law showing the average refractive index of 10 measurements (blue curve), the standard deviation (grey area) and comparison with ellipsometric measurements (dashed-dotted line). Reproduced with permission, 2018, Elsevier, ${ }^{[26]}$ (d) layer thickness measured at 10 different points (blue circles) and compared with Dektak measurements (black curve) and the associated uncertainty. Reproduced with permission, 2018, Elsevier. ${ }^{[26]}$

Finally in Figure 7(d), the results of measuring the layer thickness at 10 different points (blue circles) are given, together with the results of measurements made with a Dektak stylus profiler (black curve) and the associated measurement uncertainty, ${ }^{[26]}$ showing a very good accuracy of the local measurement technique.

We thus demonstrate that it is possible to make simultaneous measurements of both the local thickness and the refractive index (dispersion) of transparent layers greater than $500 \mathrm{~nm}$ in thickness without any prior assumptions about either of the two parameters. Certain precautions need to be taken nonetheless, such as using a closed aperture diaphragm to reduce errors in the amplitude of the measured spectrum and using a wavelength-dependent index for the reflectance spectrum model in the case of more dispersive layers. The proposed technique does not require a separate spectrometer attached to the microscope as in $^{[22]}$ and does not require knowledge of the refractive index of the layer to be measured. ${ }^{[24]}$ Model based look up tables are not required as in the first technique proposed in $^{[25]}$ and full field mapping of layer parameters is provided compared with the point measurements of the second "pupil-plane" technique proposed in the same work, although the latter has the advantage of higher accuracy due to its function as an ellipsometer.

\section{Size measurement of individual spherical particles buried in a transparent layer}

The last application of local spectroscopy using interference microscopy concerns the characterization of individual $\mu \mathrm{m}$ sized particles in buried layers. The method is based on the analysis of the backscattering spectrum of the particles, which is obtained from the spectral analysis of the interference signals. Just as with transparent layers, matching the resulting experimental backscattering spectrum of the particle with the theoretical one allows the estimation of its size and refractive index. ${ }^{[14]}$ Two situations are considered: the first for beads in a transparent layer and the second for beads in a scattering layer. 
For the first case of beads buried in a transparent layer, both simulations and real experimental measurements are made. A typical fringe signal from a bead buried in a layer is shown in Figure 8(a) with the windowed part corresponding to the front and rear fringe signals of the bead zoomed in Figure 8(b). The spectral response of spherical particles can be much more complicated than that of transparent layers, so it is essential to well define the theoretical model of the backscattering spectrum. For quite large beads, i.e. large enough so that the optical thickness is more than half the coherence length, a simple spectral response model can be derived from that used for transparent films. Difficulties arise for smaller beads as the model needs to use scattering coefficients calculated using Mie theory. ${ }^{[14]}$ If the spherical bead is large enough and assumed to be illuminated by plane waves with incident directions within a cone of $\mathrm{NA}=0.1$ (which is the case for the following results), the following simplified expression can be used to calculate the reflectance spectra of the bead: $\left|r_{\text {tot }}(k)\right|^{2}=\left(1-r_{s_{1}}^{2}(k)\right)^{2}\left[r_{f}^{2}(k)+r_{r}^{2}(k)\left(1-r_{f}^{2}(k)\right)^{2}+2 r_{r}(k) r_{f}(k)\left(1-r_{f}^{2}(k)\right) \cos \left(2 k d n_{p} \cos \theta+\phi_{f}-\phi_{r}\right)\right]$,

where $r_{f}, \phi_{f}$ and $r_{r}, \phi_{r}$ are respectively the magnitude and the phase of the Fresnel reflection coefficient for the front and rear interfaces of the particle. The terms $d$ and $n_{p}$ denote its diameter and refractive index, respectively. The coefficient $r_{s l}$ takes into account the reflection at the interface between the surrounding medium and the layer that contains the sphere. In its simplified form, this expression substantially reduces the complexity of the processing for the recovery of the size and refractive index of the structure and can also be used more broadly for different kinds of particles.
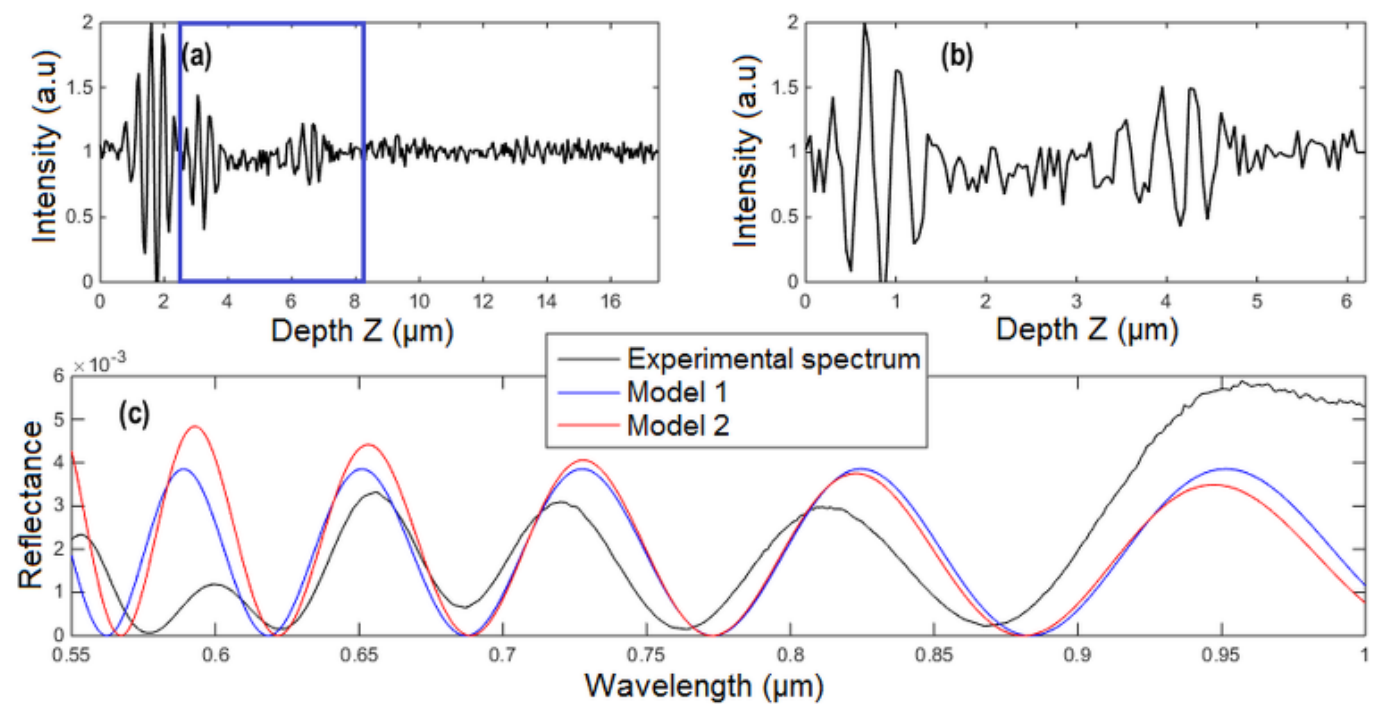

Figure 8 Measurement of the diameter and refractive index of a $2 \mu \mathrm{m}$ polystyrene bead $(n=$ 1.58) buried in gelatin ( $n=1.48$ ) (a) total interference signal of the sample, (b) windowed signal from the blue square in (a) including the fringes from the top and bottom surfaces of 
the bead, (c) comparison between the experimental backscattering spectrum (black curve) and the optimized models set by the size and the index (blue curve, Model 1) or just the size (red curve, Model 2). The results show $d=1.959 \pm 0.104 \mu \mathrm{m}$ and $n=1.579 \pm 0.028$ for $\mathrm{M}_{1}$ and $d=$ $1.957 \pm 0.031 \mu \mathrm{m}$ for $\mathrm{M}_{2}$. Reproduced with permission, 2018, Elsevier. ${ }^{[14]}$

Different samples of gelatin layers $(n=1.48)$ on microscope slides were prepared, some containing silica beads $(n=1.45, d=1.70 \mu \mathrm{m})$ and some, polystyrene beads $(n=1.58, d=2$ $\mu \mathrm{m}$ and $3 \mu \mathrm{m})$. Measurements were then performed on the Leitz-Linnik microscope with the aperture diaphragm closed down. The measured backscattering spectrum of the polystyrene beads in gelatin is plotted in Figure 8(c) (black curve). The results are then shown for two optimized models of the backscattering spectrum when using only the diameter (red curve) or both the diameter and the index (blue curve) as fitting parameters.

In the case of the polystyrene bead, the refractive index variation between the bead and the layer is small, i.e. $\sim 0.1$, and we thus estimated the SNR to be $\sim 9 \mathrm{~dB}$ after averaging. Using an optimized model set by the size and the index (Model 1), values of $1.959 \pm 0.104 \mu \mathrm{m}$ and $1.579 \pm 0.028$ are respectively obtained for the diameter and refractive index of an individual bead in Figure 8. Using just the size (Model 2), a diameter of $1.957 \pm 0.031 \mu \mathrm{m}$ is calculated. All these values match the expected ones $\left(2 \mu \mathrm{m}\right.$ and 1.58). ${ }^{[14]}$

The results of this work demonstrate that it is possible to detect and discriminate particles having a very low index contrast with the surrounding medium $(0.1$ for polystyrene beads and 0.03 in the case of silica). Low noise instrumentation devices and/or post-processing for noise reduction are nonetheless often required as the correct recovery of their characteristics depends on the signal to noise ratio in the measured interferometric signal. The particles could be discriminated between if they were separated by more than $0.8 \mu \mathrm{m}$ laterally and $2.8 \mu \mathrm{m}$ axially, limited by the lateral resolution and axially windowed resolution of the system respectively.

In order for local spectroscopy techniques to be used in biological samples, it is necessary for the technique to work on particles in scattering media. The final part of this work therefore consisted of developing the theory for characterizing beads buried in a scattering layer. Figure 9 illustrates the use of interferometry to characterize an object buried in a scattering layer, here placed at the rear face of a layer of thickness $e$ and refractive index $n$. The additional complication compared with a transparent layer is the existence of scattered rays (in blue) in addition to ballistic rays (in black). 

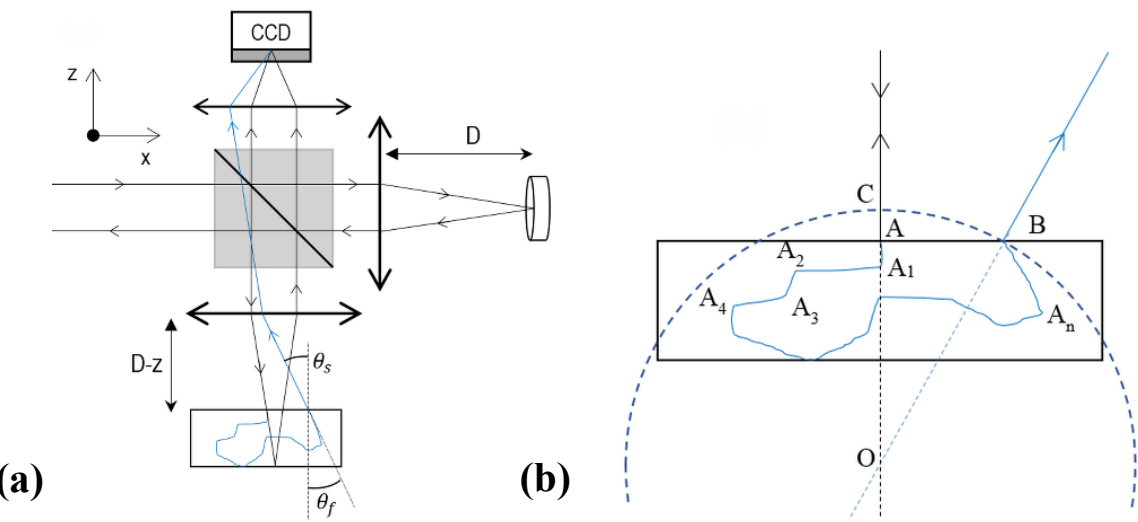

Figure 9. Using interferometry to characterize an object buried in a scattering layer (a) path difference between a ballistic (black) and a scattered (blue) ray from the emission to the collection by the camera, (b) phase shift at the output of the medium between a scattered ray (blue) and the reference ray (black). The reference mirror is in the plane defined by the surface of the sample. Reproduced with permission, 2018, Elsevier. ${ }^{[14]}$

The use of the geometrical developed approach for interference signal simulation in scattering media $^{[14]}$ for a $5 \mu \mathrm{m}$ diameter spherical particle with a refractive index $n_{p}=1.45$ located at a depth of $e=40 \mu \mathrm{m}$ within a highly scattering medium where $n=1.33$, the anisotropy factor is 0.6 and the scattering coefficient is $500 \mathrm{~cm}^{-1}$, leads to the normalized spectra marked in black in Figure 10(a). The optimization procedure using the simple backscattering spectrum model in Equation (9) does not allow the recovery of both the diameter and refractive index of the bead as the scattering is not accounted for in this model and leads to strong attenuation of ballistic light in real experiments. Nonetheless, we showed that by normalizing both the model and the simulated spectrum, assuming that the refractive index is already known and using the same optimization procedure, the diameter of the bead was estimated to be 4.998 $\mu \mathrm{m}$ (Figure 10(b)), demonstrating the effectiveness of the method even in the case of highly scattering samples.

These results of theoretical simulations of the optical properties of spherical beads in a scattering medium in order to determine their size show that such a procedure would be promising for example for disease detection in a complex biological environment as it would enable the identification of structures based on both their size and/or optical index whose modification is often an indication of health issues. 


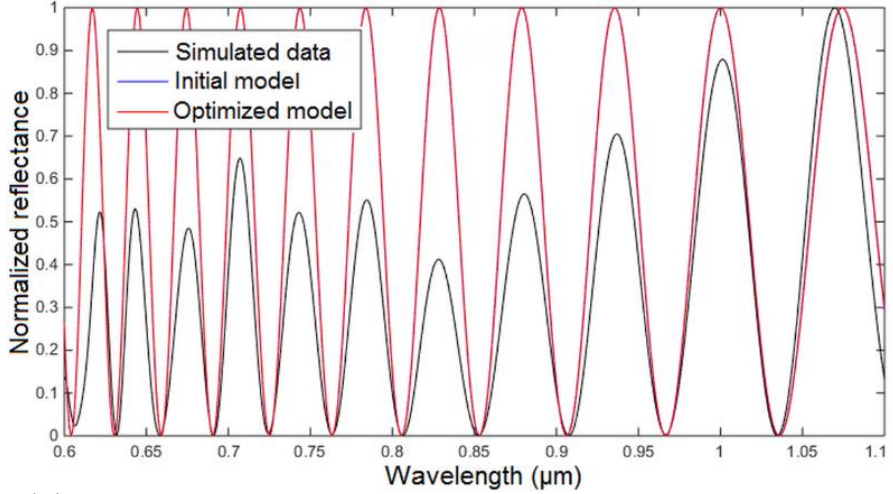

(a)

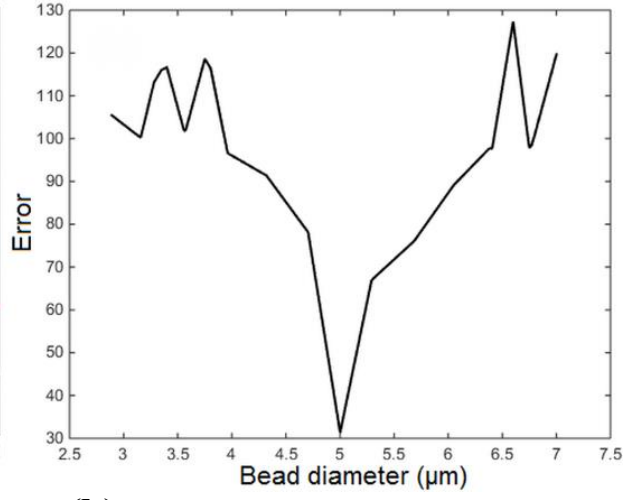

(b)

Figure 10 Theoretical estimation of the size of a $5 \mu \mathrm{m}$ diameter spherical particle with a refractive index $n_{p}=1.45$ located at a depth of $\mathrm{e}=40 \mu \mathrm{m}$ within a highly scattering medium where $\mathrm{n}=1.33$, the anisotropy factor is 0.6 and the scattering coefficient is $500 \mathrm{~cm}^{-1}$. (a) Comparison of the normalized simulated spectrum with the normalized model defined by Eq. 9 for simulated data (black curve), the real diameter of $5 \mu \mathrm{m}$ of the particle (red curve) and the diameter of $4.998 \mu \mathrm{m}$ (blue curve, hidden under red curve) obtained from the minimization of (b) the error function $\chi$. Reproduced with permission ${ }^{[14]} 2018$, Elsevier.

\section{Conclusion}

In this work we have presented the basic principles of performing local spectroscopy using interference microscopy and extended it to provide depth resolution and information on transparent layers and on particles buried in transparent and scattering layers.

Spatially resolved (XYZ) local spectroscopy has been demonstrated using interference microscopy with successful reflectance spectra on different materials in the same field of view (XY) with a lateral resolution of $0.5 \mu \mathrm{m}$ and depth-resolved reflectance spectra of materials buried under a transparent layer.

The technique has been extended to performing simultaneous measurements of both the local thickness and the refractive index (dispersion) of transparent and scattering layers above 500 $\mathrm{nm}$ in thickness without any prior assumptions about either of the two parameters. Certain precautions need to be taken nonetheless, such as using a closed aperture diaphragm to reduce errors in the amplitude of the measured spectrum and using a wavelength-dependent index for the reflectance spectrum model in the case of more dispersive layers.

Finally, more complicated measurements of the size of individual spherical particles buried in transparent layers have been performed as well as being able to discriminate between them with a spatial resolution of $0.8 \mu \mathrm{m}$ laterally and $2.8 \mu \mathrm{m}$ axially. Results demonstrate that it is 
possible to detect particles having a very low index contrast between the beads and the surrounding medium, of 0.1 for polystyrene beads and 0.03 for silica.

The results of theoretical simulations of the optical properties of spherical beads in a scattering medium in order to determine their size shows that such a procedure would be promising for example for disease detection in a complex biological environment as it would enable the identification of structures based on both their size and optical index whose modification is often an indication of health issues.

Future work is in progress to now produce spectral maps (whole images), as well as to improve the lateral resolution of local spectroscopic measurements.

\section{Acknowledgements}

The authors would like to acknowledge the financial support of this work from the University of Strasbourg and INSA Strasbourg. The authors also wish to thank the staff from the $\mathrm{C}^{3} \mathrm{Fab}$ platform for helping with the sample preparation and characterization.

\section{Conflict of Interest}

The authors declare no conflict of interest.

Received: ((will be filled in by the editorial staff))

Revised: ((will be filled in by the editorial staff)) Published online: ((will be filled in by the editorial staff))

\section{References}

[1] A. J. Huber, J. Wittborn, R. Hillenbrand, Nanotechnology 2010, 21, 235702.

[2] I. Amenabar, S. Poly, W. Nuansing, E. H. Hubrich, A. A. Govyadinov, F. Huth, R. Krutokhvostov, L. Zhang, M. Knez, J. Heberle, A. M. Bittner, and R. Hillenbrand, Nature Communications 2013, 4, 2890.

[3] S. J. Leavesley, N. Annamdevula, J. Boni, S. Stocker, K. Grant, B. Troyanovsky, T. C. Rich, D. F. Alvarez, J. Biophotonics 2012, 5(1), 67-84.

[4] A. R. Badireddy, M. R. Wiesner, J. Liu, Environ. Sci. Technol. 2012, 46, 10081-10088.

[5] J. Schmit, J. Reed, E. Novak, J. K. J. Gimzewski, Opt. A: Pure Appl. Opt. 2008, 10, 064001.

[6] P. J. de Groot, in Handbook of Optical Metrology: Principles and Applications (Ed. T. Yoshizawa), CRC Press, Boca Raton, USA 2015, Ch. 31.

[7] P. C. Montgomery, F. Anstotz, F. Salzenstein, D. Montaner, in Full-Field Optical 
Coherence Microscopy: technology and applications, (Ed. A Dubois), Pan Stanford Publishing, Singapore 2016, Ch. 11.

[8] J. L. Beverage, X. C. de Lega, M. F. Fay, in Proc. SPIE Optical Engineering \& Applications, Interferometry XVII: Techniques and Analysis (Eds. K. Creath, J. Burke, J. Schmit), 2014, 9203, 92030S.

[9] A. Akbarinia, K. R. Gegenfurtner, J. Opt. Soc. Am. A 2018, 35, B231-B238.

[10] R. D. Alcock, J. M. Coupland, Meas. Sci. Technol. 2006, 17(11), 2861-2868.

[11] A. Dubois, J. Moreau, C. Boccara, Opt. Express 2008, 16, 17082.

[12] G. Latour, J. Moreau, M. Elias, J. M. Frigerio, Opt. Comm. 2010, 283, 4810.

[13] R. Claveau, P. Montgomery, M. Flury, D. Montaner, in Proc. SPIE Photonics Europe: Optical Micro- and Nanometrology (Eds. C. Gorecki, A.K. Asundi, W. Osten), Brussels, Belgium, 2016, 9890.

[14] R. Claveau, P. Montgomery, M. Flury, Ultramicroscopy 2020, 208, 112859.

[15] A. Leong-Hoï, R. Claveau, M. Flury, W. Uhring, B. Serio, F. Anstotz, P. Montgomery, in Proc. SPIE Europe Optical Metrology, Videometrics, Range Imaging, and Applications, (Eds. F. Remondino, M.R. Shortis), Munich, Germany, 2015, 9528.

[16] A. Dubois, K. Grieve, G. Moneron, R. Lecaque, L. Vabre, C. Boccara, Appl. Opt. 2004, 43, 2874-2883.

[17] S. Marbach, C. Cordier, R. Claveau, T. Engel, P. C. Montgomery, M. Flury, in Proc. SPIE Photonics Europe Digital Forum, Optics and Photonics for Advanced Dimensional Metrology, (Eds. P. J. de Groot, R. K. Leach, P. Picart), Strasbourg, France, 2020, 11352.

[18] R. Claveau, P. Montgomery, M. Flury, Phys. Status Solidi C 2017, 14(11), 1700157.

[19] R. Claveau, P. Montgomery, M. Flury, D. Montaner, Opt. Express 2017, 25, 2021620232.

[20] P. Lehmann, S. Tereschenko, B. Allendorf, S. Hagemeier, L. Hüser, Journal of the European Optical Society-Rapid Publications, 2019, 15, 5.

[21] A. Morin, J. M. Frigerio, Applied Optics 2012, 51, 3431.

[22] S. K. Debnath, M. P. Kothiyal, J. Schmit, P. Hariharan, Opt. Express 2006, 14, 46624667.

[23] T. Guo, Z. Chen, M. Li, J. Wu, W. Fu, X. Hu, Appl. Opt. 2018, 57, 2955.

[24] S. W. Kim, G. H. Kim, Appl. Opt. 1999, 38, 5968-5973.

[25] P. J. de Groot, X. Colonna de Lega, M. Fay, in Proc. SPIE Optical Engineering + Applications, Interferometry XIV: Applications (Eds. E.L. Novak, W. Osten, C. 
Gorecki), San Diego, California, USA 2008, 7064.

[26] R. Claveau, P. Mongomery, M. Flury, G Ferblantier, Opt. Materials 2018, 86, 100-105.

[27] R. Claveau, P. Montgomery, M. Flury, in Proc. SPIE Photonics Europe, Optical Microand Nanometrology VII (Eds. C. Gorecki, A. K. Asundi, W. Osten), Strasbourg, France, 2018, 10678.

\section{TOC text and image}

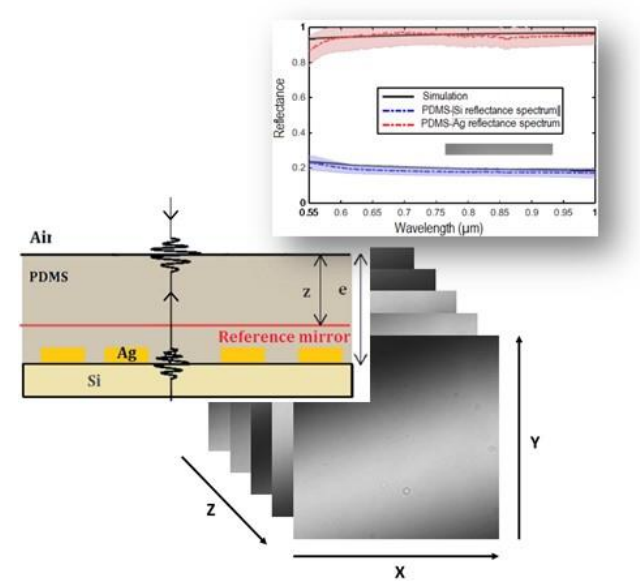

The technique of local spectroscopy using white light interference microscopy for characterizing functionalized materials is reviewed. Experimental and simulated results of spatially resolved reflectance spectra are given on different material surfaces in air and buried under a transparent PDMS layer. The technique is extended to the local measurements of refractive index and thickness of transparent layers and to the characterization of individual spherical particles buried in transparent and scattering layers with a priori information.

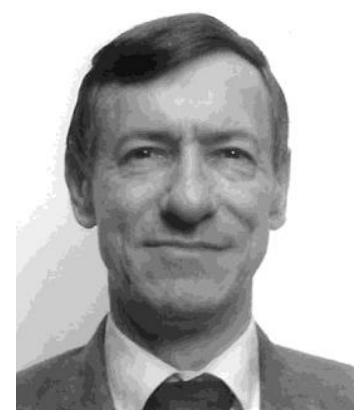

\section{Corresponding author's biography}

Paul Montgomery is a senior research scientist with the CNRS at the Engineering science, computer science and imaging laboratory (ICube) in Strasbourg, France. He is head of the D- 
ESSP (electronics and photonics) department and Deputy Director of ICube. He has over 30 years' experience in optical metrology and is currently interested in label-free far field nanoscopy and in particular local spectroscopy and microsphere-assisted interference microscopy. He is a member of SPIE, IOP and SFO. 